\title{
Strength of adhesive contacts: Influence of contact geometry and material gradients
}

\author{
Valentin L. POPOV ${ }^{1,2, *}$, Roman POHRT ${ }^{1}$, Qiang LI ${ }^{1}$ \\ ${ }^{1}$ Institute of Mechanics, Technische Universität Berlin, Berlin 10623, Germany \\ ${ }^{2}$ National Research Tomsk Polytechnic University, Tomsk 634050, Russia \\ Received: 01 April 2017 / Revised: 07 June 2017 / Accepted: 19 June 2017 \\ C The author(s) 2017. This article is published with open access at Springerlink.com
}

\begin{abstract}
The strength of an adhesive contact between two bodies can strongly depend on the macroscopic and microscopic shape of the surfaces. In the past, the influence of roughness has been investigated thoroughly. However, even in the presence of perfectly smooth surfaces, geometry can come into play in form of the macroscopic shape of the contacting region. Here we present numerical and experimental results for contacts of rigid punches with flat but oddly shaped face contacting a soft, adhesive counterpart. When it is carefully pulled off, we find that in contrast to circular shapes, detachment occurs not instantaneously but detachment fronts start at pointed corners and travel inwards, until the final configuration is reached which for macroscopically isotropic shapes is almost circular. For elongated indenters, the final shape resembles the original one with rounded corners. We describe the influence of the shape of the stamp both experimentally and numerically.

Numerical simulations are performed using a new formulation of the boundary element method for simulation of adhesive contacts suggested by Pohrt and Popov. It is based on a local, mesh dependent detachment criterion which is derived from the Griffith principle of balance of released elastic energy and the work of adhesion. The validation of the suggested method is made both by comparison with known analytical solutions and with experiments. The method is applied for simulating the detachment of flat-ended indenters with square, triangle or rectangular shape of cross-section as well as shapes with various kinds of faults and to "brushes". The method is extended for describing power-law gradient media.
\end{abstract}

Keywords: adhesion; boundary element method (BEM); flat-ended indenters, gradient media

\section{Introduction}

"Adhesion" is a term which is used for describing different phenomena depending on the branch of science and technology [1]. In the present paper we understand under "adhesion" the relatively weak, so-called van der Waals interaction which acts between any electrically neutral bodies [2,3]. These forces cause "sticking together" of two solids when they are brought into a contact. In everyday life, adhesive forces can be easily seen in a contact of a very soft elastic material (an elastomer or a jelly) and a smooth solid body. It appears also in a contact of two solids divided by a soft layer as in various types of "stickers" and sticking plasters which are widely used in domestic, industrial and medical applications [4]. Adhesion is used by many groups of living organisms as a mechanism allowing them to attach to various kinds of surfaces [5]. The most famous example of an extremely effective adhesion device is gecko feet which inspired numerous studies of adhesion in the last decade [6]. Adhesion plays an important role in cell mechanics and proliferation [7]. Adhesion is of high technological interest as it is the basis for huge industries of adhesive bonding or reversible "sticking". However, it can also be an annoying and disturbing

* Corresponding author: Valentin L. POPOV, E-mail: v.popov@tu-berlin.de 
factor, e.g., preventing a rapid opening of elastomer valves.

Even when the van der Waals forces are much weaker than covalent interactions, the adhesive stress is high enough (on the order of magnitude of $10 \mathrm{GPa}$ [8]) to provide high strength adhesive bonding in all the cases when it is possible to create an intimate contact on the atomic scale. As stated by Kendall [9], "solids are expected to adhere; the question is to explain why they do not, rather than why they do!" The reason for the obvious weakness of macroscopic adhesion in everyday-life is in most cases either a stress concentration on the boundary of a contact, a crack or the roughness of surfaces that hinders the intimate contact of the two bodies. The influence of roughness was in focus of adhesion studies over many years. Now it is well understood under which conditions the roughness can "kill" the adhesion $[10,11]$ and when it can even enhance it [12].

However, not only roughness defines the adhesive contact. Even in the presence of perfectly smooth surfaces, geometry can come into play in form of the macroscopic shape of the contacting region. Think for instance of medical plasters which have sharp rectangular shape. It is commonplace experience that they tend to detach at their corners first. With rounded edges instead, they hold a lot better, so their sticking capability depends on the contour. This influence of the macroscopic shape of the contact area remained till now out of focus of the research of adhesion. In the present paper we will report an experimental and numerical study on a related model problem. We will consider rigid cylindrical indenters with flat but oddly shaped face in contact with a soft, adhesive counterpart (as schematically shown in Fig. 1). Despite the apparent simplicity of the system, it shows non-trivial and very puzzling behavior!

In mathematical sense, an adhesive contact is equivalent to a crack. In their paper from 1971 [13] - maybe the most famous paper on the theory of adhesive contacts-Johnson, Kendall and Roberts wrote: "the approach followed in this analysis, is similar to that used by Griffith in his criterion for the propagation of a brittle crack." They realized that the adhesive contact is nothing but an "inverted crack"

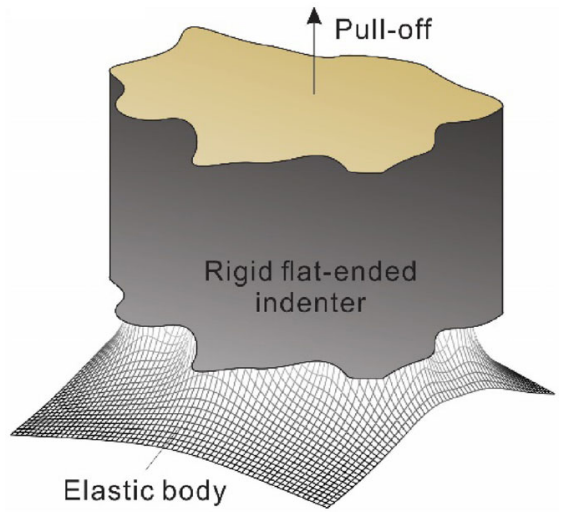

Fig. 1 We consider adhesive contacts of rigid indenters with flat but oddly shaped face with elastic half-space.

and repeated the Griffith analysis for this particular geometrical configuration. In the early 1970s, models in fracture mechanics were already well developed. Because these could be transferred, the theory of adhesion advanced rapidly [14]. Almost at the same time, Cruse, Rizzo and Brebbia introduced the method they called boundary element method (BEM) [15]. However, early formulations of BEM [16, 17] suffered from the problem of inverting the fully populated matrices. Only after algorithms based on fast Fourier transformation were introduced, the BEM became an efficient method. The incorporation of the Griffith criterion in these modern implementations of BEM was first done in 2015 by Pohrt and Popov [18]. The same idea was independently proposed in 2016 by Hulikal et al. [19]. Recently, Rey et al. [20] suggested an alternative approach to the adhesive BEM, which is based on the minimization of the total energy. In the following sections we use the adhesive BEM as described in Ref. [18] for the simulation of adhesion in contacts with various macroscopic shapes and compare with experimental data. We start in Section 2 with general considerations of the adhesion of rigid flatended indenters and introduce notions and definitions used later in the paper. We then describe in Section 3 how the Griffith' approach is implemented in the modern boundary element programs for numerical simulation of adhesive contacts in the case of homogeneous media. In the same section, the validation of the method through comparison with known analytical solutions is presented. Sections 4 to 9 are devoted to numerical analysis of the detachment of flat-ended 
stamps of various shape of cross-section. Section 10 describes experiments with adhesive contacts of flatended indenters. In Section 11, the generalization of the adhesive BEM to graded materials is presented followed by conclusions in section 12 .

\section{General theoretical considerations}

\subsection{Flat-ended cylinder with circular cross-section}

In the present paper we consider adhesive contacts between variously shaped bodies, but the main attention is paid to "flat ended stamps". Here we introduce some notations which will be needed for discussion in subsequent sections. We start our discussion with recapitulation of the classical problem of adhesion of a flat-ended cylinder with circular cross-section solved by Kendall in 1971 [21]. Let us consider an adhesive contact between a rigid body with plane surface and an elastic half-space in a state with a contact radius $a$ and the indentation depth $d$. The differential stiffness of a contact with the radius $a$ is equal to

$$
k=2 E^{*} a
$$

where $E^{*}=E /\left(1-v^{2}\right)$ is the reduced modulus of elasticity, $E$ is the Young's modulus and $v$ the Poisson ratio [8]. The elastic energy stored in the medium is equal to $U_{\mathrm{el}}=(1 / 2) k d^{2}=E^{*} a d^{2}$ and the adhesion energy $U_{\mathrm{ad}}=-\gamma_{12} \pi a^{2}$, where $\gamma_{12}$ is the work of adhesion per unit area. Thus, the total energy of the system is equal to

$$
U_{\mathrm{tot}}=U_{\mathrm{el}}+U_{\mathrm{ad}}=E^{*} a d^{2}-\gamma_{12} \pi a^{2}
$$

At a given $d$, this function has a maximum at

$$
a_{\mathrm{c}}=\frac{E^{*} d^{2}}{2 \pi \gamma_{12}}
$$

This maximum is the only equilibrium state and it is non-stable. If the initial value of $a$ exceeds the critical value defined by Eq. (3), the contact spreads to the infinity (or, if the plane is finite, to the maximum possible radius). For any initial $a$ smaller $a_{\mathrm{c}^{\prime}}$ it shrinks to zero, and the bodies lose the contact. For a cylindrical indenter with finite radius $a$, Eq. (3) gives the relation between the radius and the critical value of $d$ in the moment of detachment: $a=E^{*} d_{c}^{2} /\left(2 \pi \gamma_{12}\right)$, whence

$$
d_{c}=-\sqrt{\frac{2 \pi \gamma_{12} a}{E^{*}}}
$$

where we take the negative solution, as only in this case detachment is (geometrically) possible. The normal force in this critical state is

$$
F_{\mathrm{N}}=k d_{\mathrm{c}}=-2 E^{*} a \sqrt{\frac{2 \pi \gamma_{12} a}{E^{*}}}=-\sqrt{8 \pi E^{*} \gamma_{12} a^{3}}
$$

The corresponding "force of adhesion" $F_{\mathrm{A}}$ is just the absolute value of this force:

$$
F_{A}=\sqrt{8 \pi E^{*} \gamma_{12} a^{3}}
$$

which reproduces the solution of Kendall [21].

It is interesting to note that in the moment of detachment the elastic energy $U_{\mathrm{el}, \mathrm{c}}$ is equal to

$$
U_{\mathrm{el}, \mathrm{c}}=2 \pi a^{2} \gamma_{12}
$$

while the energy which is needed in order to create the surface area $\pi a^{2}$ should be only

$$
\Delta U_{\mathrm{ad}}=\pi a^{2} \gamma_{12}
$$

Thus, half of the external work used for detaching the punch is not for creating the surface, but will eventually dissipate in elastic waves emitted into the elastic body.

\subsection{Flat-ended cylinder with arbitrary cross-section}

The above analysis can be easily generalized for adhesion of flat-ended indenters whose cross-sections are not a circle but are compact and have no sharp corners (as, e.g., a square or a triangle with rounded corners or similar). In such cases, the contact stiffness is given approximately by

$$
k=\beta \cdot 2 E^{*} \sqrt{\frac{A}{\pi}}
$$

where $A$ is the cross section area of the indenter and $\beta$ is a numerical factor on the order of unity. As shown numerically in Ref. [22], for a square crosssection $\beta=1.021$. For any shape with rounded corners, 
this coefficient is even smaller. Thus, with an accuracy of about $2 \%$ we can use the Eq. (9) with $\beta=1$. In this approximation, Eq. (2) takes the form

$$
U_{\mathrm{tot}} \approx E^{*} \sqrt{\frac{A}{\pi}} d^{2}-\gamma_{12} A
$$

It has one single maximum at

$$
A_{\mathrm{c}} \approx \frac{1}{\pi}\left(\frac{E^{*} d^{2}}{2 \gamma_{12}}\right)^{2}
$$

At the given cross-section area $A$, this equation determines the critical approach $d_{\mathrm{c}}$ :

$$
A \approx \frac{1}{\pi}\left(\frac{E^{*} d_{\mathrm{c}}^{2}}{2 \gamma_{12}}\right)^{2}
$$

It is easy to see that Eqs. (4) and (5) remain valid if the "effective radius" $\sqrt{A / \pi}$ is used instead of $a$. Thus, the estimations of the adhesive force and critical indentation depth are given by the equations

$$
F_{\mathrm{A}, \text { upper }}=\sqrt{8 \pi E^{*} \gamma_{12}(\sqrt{A / \pi})^{3}}
$$

and

$$
d_{\text {c,upper }}=-\sqrt{\frac{2 \pi \gamma_{12} \sqrt{A / \pi}}{E^{*}}}
$$

We have used index "upper" as the above values give the upper bound of the adhesive stress and distance. All stamps with a "near-circle" cross-section will detach similar to a cylindrical punch: Detachment occurs (almost) at once, at latest when the cross-section area and the approach satisfy Eq. (11). This however, is not valid for more complicated shapes. We will see that for complicated shapes the detachment generally occurs not instantaneously.

At this point let us introduce some notations which we will use in the further analysis. For characterizing a particular shape we will use the so-called "Holmradius" $a_{\mathrm{H}}=a_{\mathrm{H}}(\{A\})[23]$. Note that $a_{\mathrm{H}}(\{A\})$ is not a function of the contact area but a functional of the complete contour of the contact, which is stressed by using curly brackets $\{\cdot\}$. The Holm-radius represents the radius of an equivalent circle having the same contact stiffness as the original shape. For a circle $a_{\mathrm{H}}=\sqrt{A / \pi}$ is just the radius of the indenter. In general case

$$
a_{\mathrm{H}}(\{A\}) \geq \sqrt{A / \pi}
$$

Using the notion of the Holm-radius, Eq. (2) can be rewritten as

$$
U_{\text {tot }}=E^{*} a_{\mathrm{H}}(\{A\}) d^{2}-\gamma_{12} A
$$

while the normal force is defined as

$$
F=2 a_{\mathrm{H}}(\{A\}) E^{*} d
$$

Formally, the stability conditions for a particular contact configuration can be found by solving the variational problem of minimizing the energy functional (16). The explicit analytical form of the functional $a_{\mathrm{H}}(\{A\})$ is not known. Therefore, we will conduct further studies using numerical methods.

However, it is possible to give analytically the upper and lower bounds of the interval in which the main part of the detachment process occurs. As we have seen, within an accuracy of a few percent, the detachment process must start at the distance $d_{\mathrm{c}}$ satisfying the condition (12) at the latest. According to the simple estimation, the detachment should occur at once. Equation (13) gives the upper bound for the force of adhesion. However, due to the non-circle form, there exist a possibility that the detached region will spread in a non-circular fashion so that the first term in Eq. (16) dominates the second one. The possibility of this adjustment will be completely exhausted as soon as the contact configuration reaches the incircle of the cross-section, whose radius we denote as $a_{\text {incircle }}$. After achieving this configuration, no equilibrium is possible as this circle has definitely under-critical area. The corresponding normal force in this state represents the lower bound of the adhesion force possible for the given cross-section:

$$
F_{\mathrm{A}, \text { lower }}=\sqrt{8 \pi E^{*} \gamma_{12} a_{\text {incircle }}^{3}}
$$

In this state, the approach is given by

$$
d_{\text {clower }}=-\sqrt{\frac{2 \pi \gamma_{12} a_{\text {incircle }}}{E^{*}}}
$$




\section{Numerical simulation of adhesion}

The Griffith' approach to cracks [24] played a prominent role in materials science. His idea was to consider the balance of elastic energy which is released due to a small change in the position of the crack tip and the work of adhesion which is needed to create new free surfaces-instead of a much more complicated stress analysis. The energy-based approach is especially favorable in the case of cracks and adhesive contacts as it allows avoiding the handling of stress singularities at the crack tip. In the preceding section we already used the Griffith energy balance approach to derive Kendall's result for a circular cylinder. Below we discuss briefly the main idea of the Griffith' energy based approach implemented in the boundary element method (BEM) [18].

Let us consider the simplest discretization of the contacting surfaces consisting of square elements with the side length $\Delta$ as shown in Fig. 2. The complete procedure of BEM for non-adhesive contact is described, e.g., in Refs. [25, 26]. In each calculation iteration of the BEM, the stress and displacement of each particular discretization element are determined and it is decided if the element should still remain in contact. For non-adhesive contacts this is the case as long as the pressure remains positive. In an adhesive contact, pressures may become negative and thus a more elaborated rule of detachment is needed. In Ref. [18], Pohrt and Popov suggested to make the decision about detachment of a single element based on the Griffith' energy criterion: the element will detach if the energy released by its detaching exceeds the work

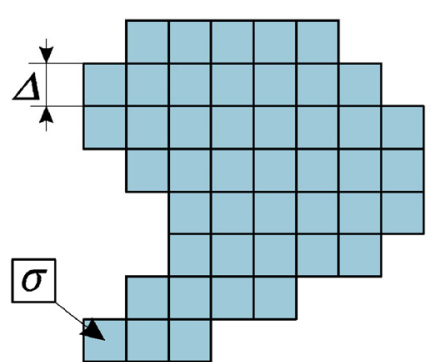

(a)

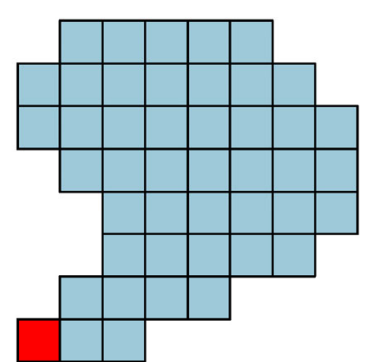

(b)
Fig. 2 In each calculation step, stress in each particular discretization element is determined. If the stress $\sigma$ in a given element at the boundary of the contact area exceeds the critical value (22), it is "detached" and the stress in this element is set zero. of adhesion. Following that, we can obtain a stress criterion.

The drop of normal stress results in a decrease of the elastic energy [18] (see also Ref. [8], 2nd edition, Chapter 19):

$$
\Delta U_{\mathrm{el}}(\tau)=\kappa \frac{\sigma^{2}}{E^{*}} \Delta^{3}
$$

with

$$
\kappa=\frac{2}{3 \pi}\left(1-\sqrt{2}+\frac{3}{2} \log \left(\frac{\sqrt{2}+1}{\sqrt{2}-1}\right)\right) \approx 0.473201
$$

The element is in the state of indifferent equilibrium if the change of elastic energy is equal to the work of adhesion needed for creating the free surface with the area $\Delta^{2}, \Delta U_{\text {adh }}=\gamma_{12} \Delta^{2}$, or with Eq. (20), $\kappa \sigma^{2} \Delta^{3} / E^{*}=$ $\gamma_{12} \Delta^{2}$, where $\gamma_{12}$ is the work of adhesion per unit area. For the critical detachment stress we obtain

$$
\sigma_{\mathrm{c}}=\sqrt{\frac{E^{*} \gamma_{12}}{0.473201 \cdot \Delta}}
$$

This is a local, mesh-dependent detachment criterion. It is local in the sense that the critical stress does not depend on the stress in any other parts of the contact. Mesh dependent means that the detachment criterion explicitly depends on the mesh-size. If the tensile stress in one of the elements at the boundary of the contact area is $\sigma$ and this element will detach, then the stress decreases from $\sigma$ to zero (the element is shown red in Fig. 2(b)).

The algorithm for a pull-off simulation of adhesive contact is the following. The indenter is initially pressed into the elastic half space to some depth without consideration of adhesion, which results in a surface displacement and some contact area. Now the indenter is pulled off in incremental steps. In each step, the contact area is first considered unchanged, and the stress corresponding to the change of surface deformation is calculated in all points of the calculation grid in the contact area as well as displacements outside the contact area. For this sake, the procedure of non-adhesive BEM described in Ref. [25] is used. Then the stress criterion is checked: all elements whose tensile stress exceeds the criterion are separated from the contact, and a new contact area is obtained. 
With this new contact area the stress distribution is recalculated. The procedure is repeated until the stresses in all elements are below the detachment condition. Finally, the normal force can be calculated by integrating the pressure, and the simulation continues to the next pull-off step.

The above adhesive BEM formulation was validated by comparison with known exact analytical solutions as, e.g., flat cylindrical punch [19], parabolic bodies [13] or cone [27], and also passed usual tests of independence of mesh size and orientation of the discretization network [28]. Results of test simulations are illustrated in Fig. 3.

The presented set of simulation results confirms that:

(a) The numerical method reproduces with high precision the known analytical solutions.

(b) Neither the macroscopic force-approach dependence nor the contact configuration does depend on the mesh size.

(c) The simulation results do not depend on the grid orientation.

(d) The square numerical grid has no influence on the axial symmetry of the simulated problem: The simulated contact areas remain exactly circular in spite of the different symmetry of the simulation grid.

Other classes of exact or "asymptotically exact" solved problems are the two-dimensional or "quasitwo-dimensional" problems (as, e.g., a contact of a torus with an elastic half-space). These analytic solutions were also used for testing the above adhesive BEM (see details see in Ref. [29].)

\section{Arbitrary contact shapes: Numerical simulation}

In Section 4, we use the above adhesive BEM [18] for simulation of contacts of flat-ended stamps having
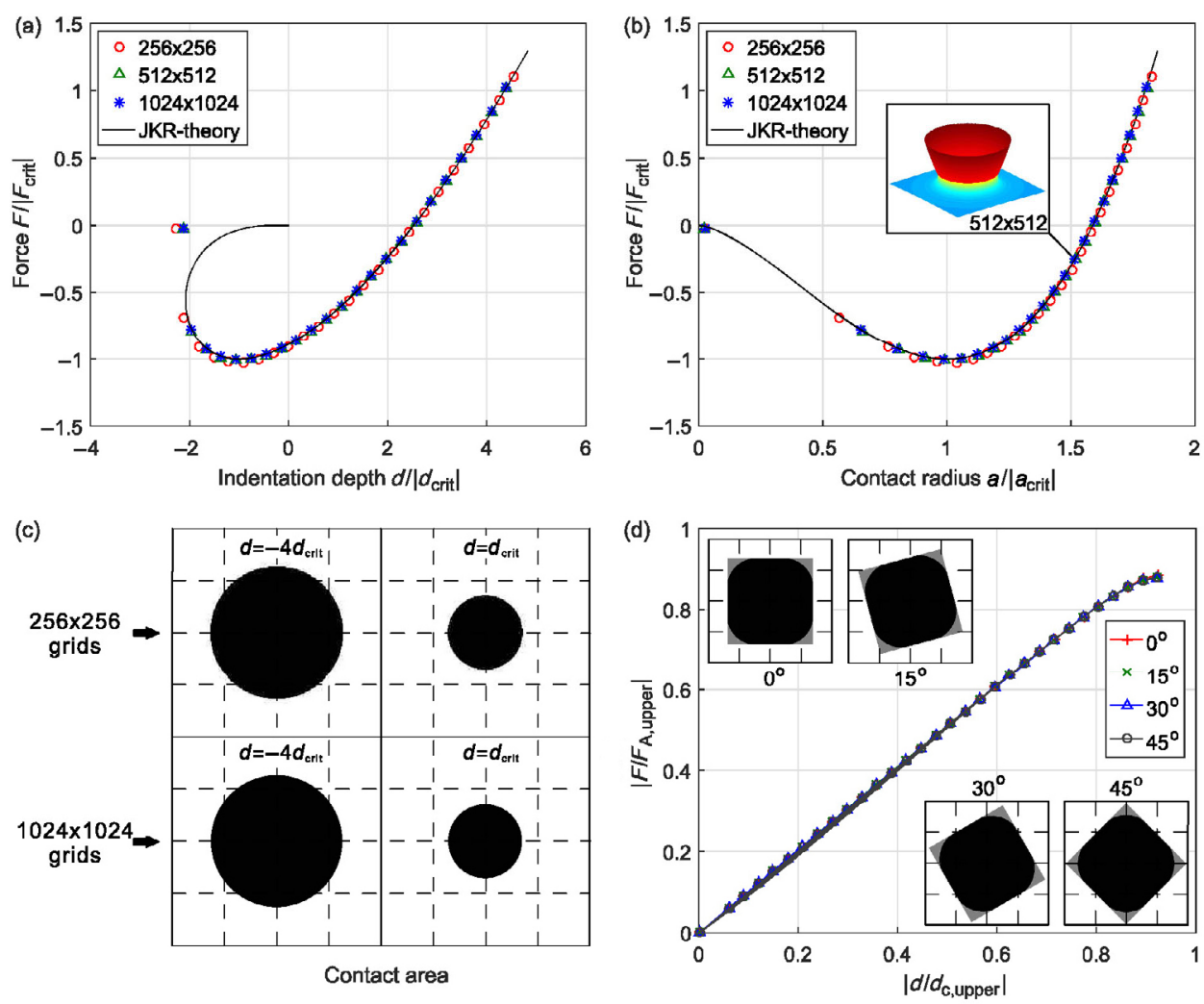

Fig. 3 Comparison of a BEM simulation of the adhesive contact between a parabolic indenter and elastic half-space (JKR-problem) and the analytical solution with (a) force-vs-approach and (b) force-vs-contact-radius relations for three different mesh sizes. (c) Contact areas at four selected approaches illustrating the ideally circular form of contact. (d) Adhesive contact of a square punch in different orientations with $512 \times 512$ grid points (dashed lines indicate the orientation of meshing grids), illustrating that neither the contact form not the force-approach-relations do depend on the grid orientation. 
non-circle cross-section shape. We simulate the flat adhesive contact for a series of compact shapes. With controlled pull-off-distance $d$ we found the contact area $A$ and the corresponding elastic force as a function of $d$. Of course, the complete stress and displacement fields are the necessary "byproducts" of the simulation.

\subsection{Convex cross-sections}

Let us start with simply-connected shapes such as that of a square or triangle. In this case, a rough picture of the detachment process is given by the approximation described in Section 2.2: With increasing distance between the bodies, the force should first increase linearly up to the critical value (14) and then drop abruptly to zero. In reality we expect not an abrupt but a "rapid" decrease.

Numerically calculated force-distance dependencies are presented in Fig. 4. The force and the distance are here normalized by the critical values of the "upper bound" (13) and (14). The quality of the approximation and the deviations can thus be immediately seen. Numerical results confirm the general picture predicted by the simple approximation: the force increases almost linearly and then drops to zero. While for circular

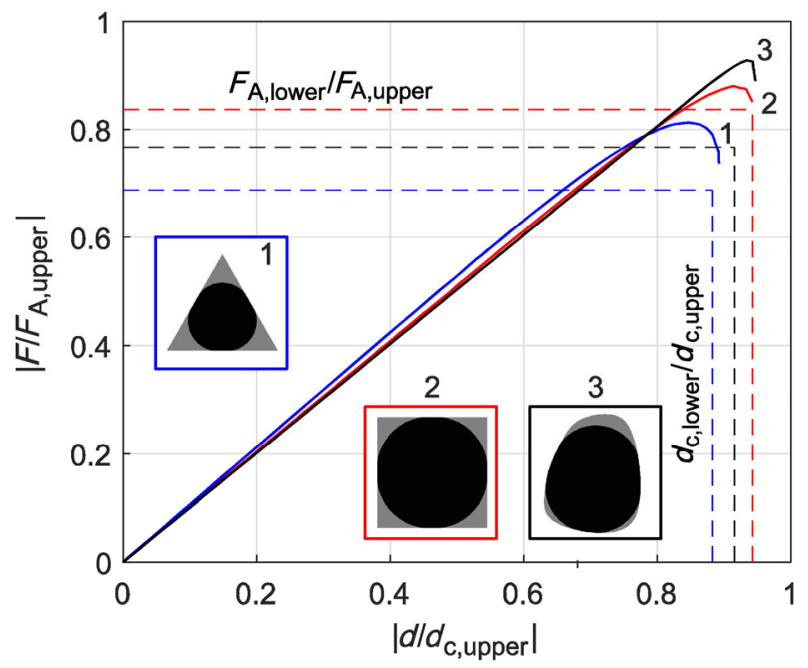

Fig. 4 Force-distance dependencies for simply connected convex profiles: triangle, square, an "arbitrary" shape. The very rough picture in this case is similar to that of a circular cylinder: the normal force first increases linearly with the distance between bodies and then drops sharply. However, the transition from the linear increase to the complete detachment now takes some (small) interval of detachment distances. The state corresponding to the incircle of the corresponding shape is shown with dashed lines. cylinders it drops at once, other forms show some prior decrease in the force. The maximum force of adhesion comes very close to the predicted one (the maxima of all curves approach the value " 1 " in dimensionless units).

\subsection{Concave cross-sections with outstanding sharp parts}

From Fig. 4 one can already see that the deviations from abrupt detachment become larger with the increase of the deviation of the shape from a circle. They become even more pronounced in the case when the indenter shape has sharp outstanding parts like the star shown in Fig. 5. In this case, partial detachment starts early at sharp ends and propagates inwards. After achieving the maximum, the force starts decreasing with further increase of the distance between the bodies. The last stable configuration is very close to
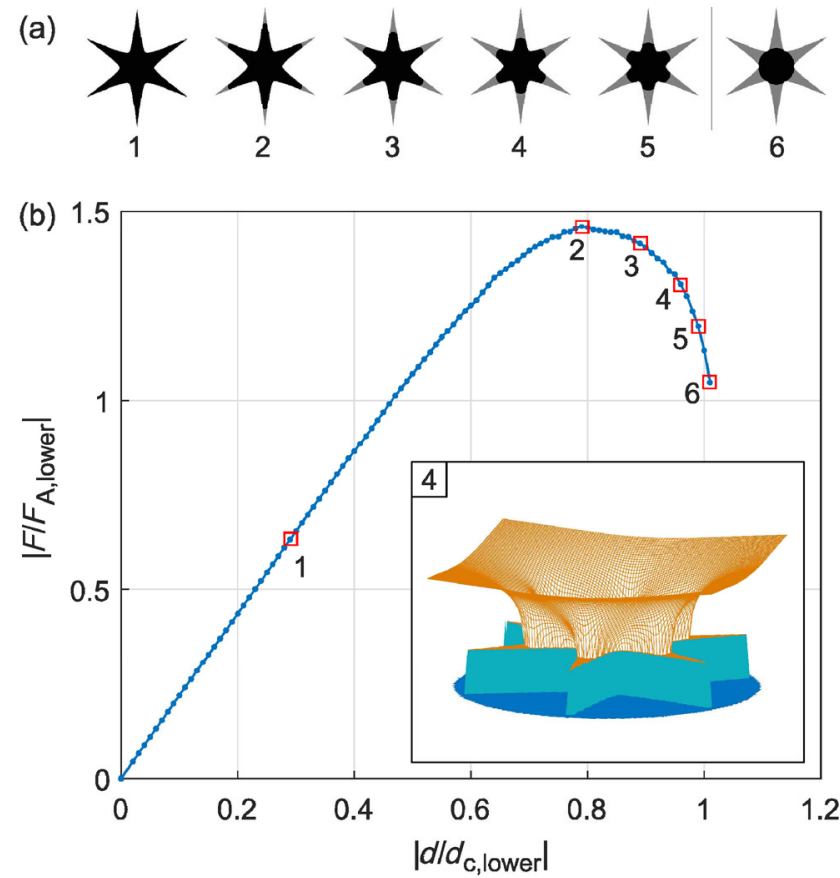

Fig. 5 Detachment process of a flat-ended indenter with the crosssection in form of a "star". (a) A series of contact configurations: grey color shows the initial shape of the indenter and black color the remaining contact area. The detachment starts at the pointed ends of the star and spreads inwards up to a state which is close to the incircle of the shape. (b) Dependence of the absolute value of the normal force on the approach. The force is normalized to the low bound value (18) and the approach to the corresponding value (19). Subplot of (b): Three-dimensional "snap-shot" of the surface of the elastic half-space at a moment of partial detachment. 
the incircle of the shape (see Fig. 5(a)). In the subplot Fig. 5(b), the dependence of the normal force on the distance is shown. Note that in this plot the force and the distance are normalized to the values corresponding to the incircle (normalization differs from that used in Fig. 4). The last stable state approaches the value "1" both for the force and distance which in normalized units corresponds to the incircle.

\subsection{General discussion of detachment of arbitrary macroscopically isotropic shapes}

Let us discuss the detachment of arbitrary complicated shapes, like those shown in Fig. 6. While at the present time a formal formulation of the stages of detachment process is not available, the general rules can be formulated at least qualitatively:

1. The detachment process tends to start at the points having the largest distance from the center of the profile and at the sharp corners.

2. While the outer parts may already be completely detached, the remaining part, which is still adhering, will provide some resistance until aproaches the incircle of the shape.

3. Small heterogeneities such as holes and other small defects have no pronounced influence on the process of detachment. In particular, the detachment rarely starts from the inner discontinuities of the shape.

This last point is interesting with respect to the problem of influence of defects and damages on the

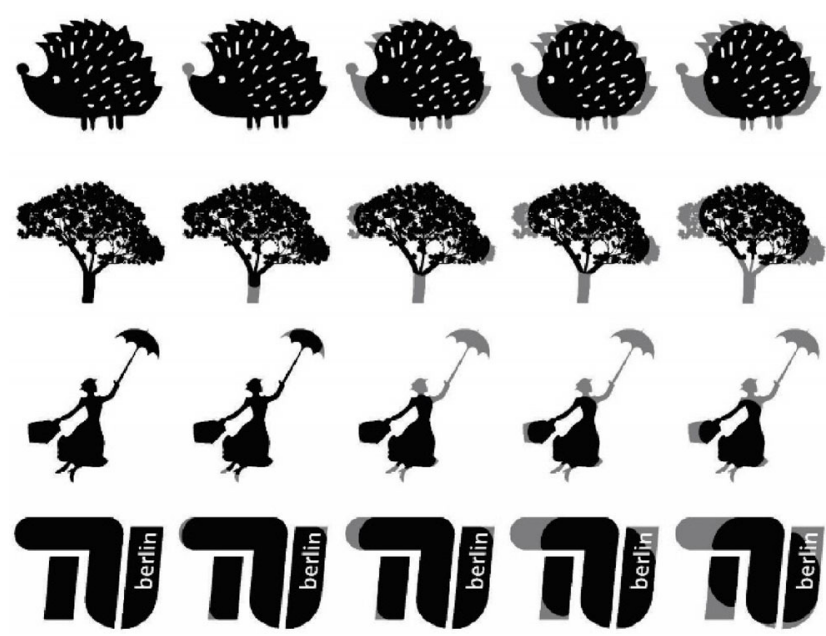

Fig. 6 Decreasing contact areas during the detachment process for a series of flat-ended indenters. general strength of an adhesive contact. In the next section, we study this question in more detail.

\section{Influence of internal discontinuities of the contact shape}

Small discontinuities at the face of the flat-ended indenter apparently have no essential influence on the adhesive strength. This is related to the fact that the strength of the contact is determined by the interplay of elastic energy (determined mostly by the stiffness of the contact) and the work of adhesion, which is proportional to the contact area. Small discontinuities do not influence the overall stiffness of the contact, and their influence on the contact area is proportional to the area of the discontinuity. This property can be seen in the curves depicted in Fig. 7 .

One can easily see that the V-shaped damage has practically no influence on the overall behavior. Even in the direct vicinity of the damage line there are almost no distinctions in the way that region is detached. One can suggest the following very rough estimation of the influence of damage on the adhesive strength. Assuming that the damage does not change the stiffness (which is governed by the outer bounds of $A$ ) and the damage changes the contact area in proportion $A_{\text {real }}=\xi A$, where $\xi$ is the filling factor of the damage,

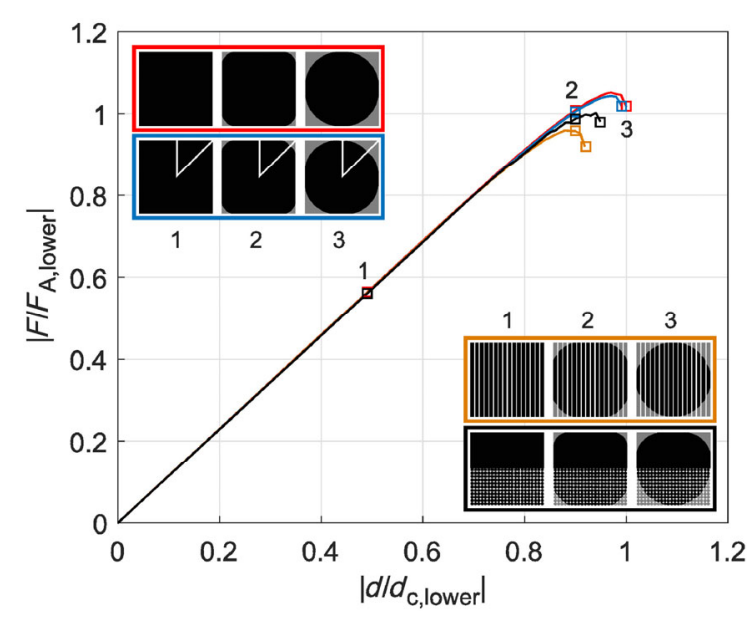

Fig. 7 Contact configurations and force-distance dependencies for a series of squares containing different kinds of faults. The plain square indenter is given as a reference. It is compared with the same square having discontinuities in form of a "V", a series of vertically oriented line-discontinuities as well as line discontinuities filling only the half of the square. 
we can rewrite Eq. (10) in the form

$$
U_{\mathrm{tot}} \approx E^{*} \sqrt{\frac{A}{\pi}} d^{2}-\gamma_{12} \xi A
$$

which differs from Eq. (10) only by replacing the work of adhesion $\gamma_{12}$ by the effective work of adhesion $\xi \gamma_{12}$. Equation (13) for the upper limit of the adhesive force will be changed to give

$$
F_{\mathrm{A}, \text { upper }}=\sqrt{8 \pi E^{*} \xi \gamma_{12}(\sqrt{A / \pi})^{3}}
$$

Thus, the force of adhesion will be roughly proportional to the square root of the filling factor $\xi$.

\section{Adhesion of brushes}

An interesting and important case are brush-like structures: A series of cylindrical, flat-ended columns distributed in some limited area. The discussion of brushes is interesting in particular in the context of contact splitting which is often believed to enhance adhesion. We will show that in our model problem, the splitting alone never enhances adhesion.

Here we present qualitative results for square bruhes. The results were obtained for regular, random and mixed brushes with columns of various shape and distribution (see Fig. 8(a)). While the details of the contact shapes and force-distance dependencies may slightly depend on the particular brush, their general properties are very robust: the sequence of the contact shapes detaching is always similar to that of a continuous square (Fig. 8(b)), and the force-distance dependencies almost collapse to a single linear dependence of the force of adhesion and on the square root of the filling parameter $\xi$ as defined in the Section 5. This master curve shows that the concept of effective surface energy introduced in Section 5 is reasonably applicable.

\section{Profiles with nearly constant normal force}

The fact that for complicated shapes the normal force can both increase and decrease with detachment distance, puts the question if it is possible to design a shape providing a particular desired behavior, e.g., a constant normal force independent on distance. (a)

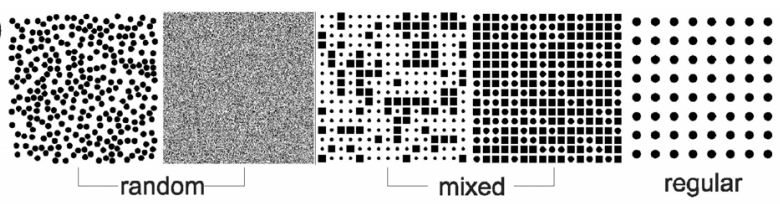

(b)
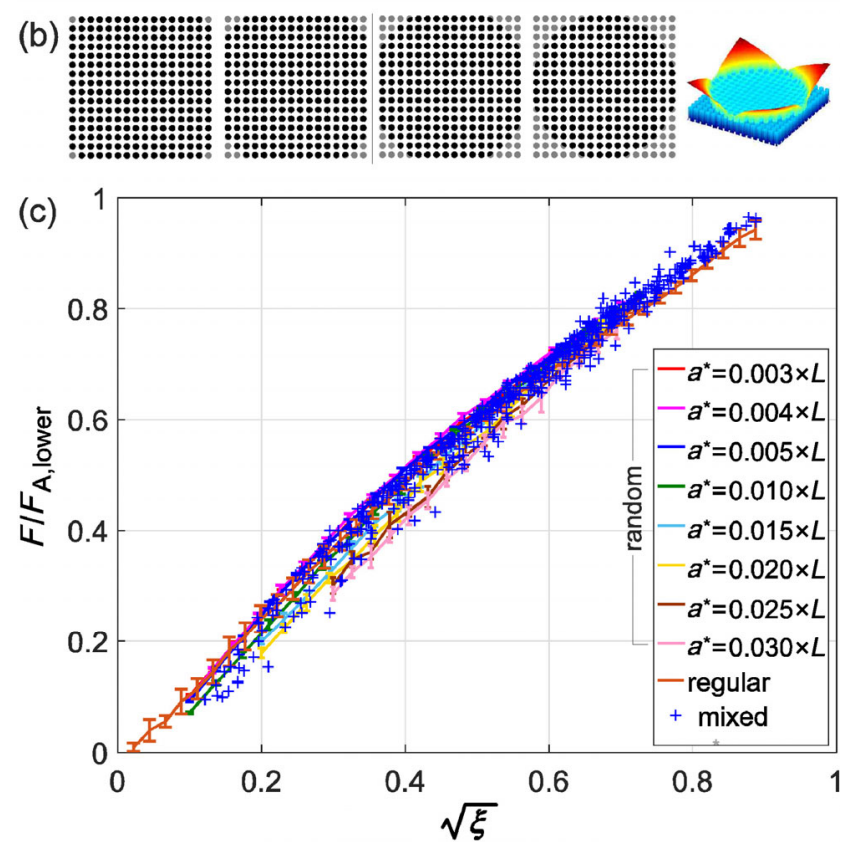

Fig. 8 (a) Shapes and distributions of spikes in a square brush studied here. (b) Consecutive stages of the contact configuration for increasing detachment distance and particular shape of the surface of the elastic counterpart at one of the displayed stages. (c) Simulation points for various brushes and sizes of spikes: All points collapse approximately to a single master curve which is approximately a linear function of the square root of the filling factor $\xi$. It is given approximately for all cases by Eq. (24). $a^{*}$ is the radius of the spikes and $L$ is the side length of square. The force is normalized to the value $F_{\mathrm{A} \text {,low }}$, corresponding to the radius $a_{\text {incircle }}$ of the complete square.

The existence of profiles with a constant normal force can also be deduced from the example of brushes. Let us consider an indenter which filling parameter $\xi$ is a function of the radius $r$. Assuming that the discontinuities of the shape do not influence the stiffness, we can write the total energy as

$$
U_{\mathrm{tot}} \approx E^{*} a d^{2}-\gamma_{12} \int_{0}^{a} 2 \pi r \xi(r) \mathrm{d} r
$$

The equilibrium state is determined from the requirement of the minimum of the total energy:

$$
\frac{\partial U_{\mathrm{tot}}}{\partial a} \approx E^{*} d^{2}-\gamma_{12} 2 \pi a \xi(a)=0
$$


The relation between $d$ and $a$ is thus given by

$$
d=\sqrt{\frac{\gamma_{12} 2 \pi a \xi(a)}{E^{*}}}
$$

The normal force in this state is given by

$$
F_{\mathrm{N}}=2 E^{*} a d=\sqrt{8 \gamma_{12} E^{*} \pi a^{3} \xi(a)}
$$

It is independent of $a$ if $a^{3} \xi(a)=$ const or

$$
\xi(a)=\mathrm{const} / a^{3}
$$

Any structure showing in some interval of $a$ this dependence of the filling parameter on the radius should provide an approximately constant adhesive force. For example, one can realize such structure by designing a star with rays having in some interval the thickness which is inversely proportional to the second power of radius. In Fig. 9, we present a spiral which was designed to show this dependence in the outer arms.

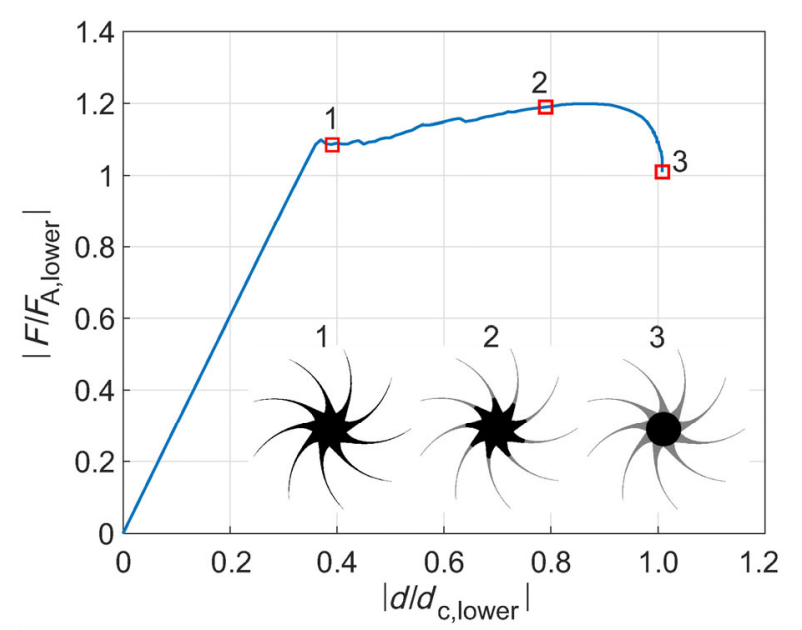

Fig. 9 Detachment process of a flat-ended indenter with the crosssection in form of a "star" having "rays" which satisfy Eq. (29). In this case, a constant pulling force is predicted in the whole range where this dependence is valid.

\section{The role of filling parameter}

Simulating the detachment of various indenter shapes has revealed a simple rough picture behind it, which we would like to discuss briefly at this point. If the dimensions of the shape are not clearly dominated by one of the in-plane-orientations (as the most shapes considered in this paper) then the detachment can be qualitatively understood in terms of the average filling parameter $\xi$. During the detachment process the remaining contact area contracts inwards and can be assigned an effective radius radius $a$. The stiffness of this contact area is almost independent from the structure of faults in the shape and can be approximated by the contact stiffness of the complete circle with the same radius: for homogeneous media, $k=2 E^{*} a$. On the other hand, the change in adhesive energy is determined by the product of the change of the contact area and the filling factor $\xi$. The approximate total energy is thus generally given by Eq. (25). The equilibrium radius $a$ is given by requiring that the energy acquires a minimum: Eq. (26). However, this equilibrium condition can only be realized if the equilibrium is stable, thus, additionally the condition $\partial^{2} U_{\text {tot }} / \partial^{2} a>0$ must be satisfied. From Eq. (26), it follows

$$
\frac{\mathrm{d}}{\mathrm{d} a}(a \xi(a))<0, \quad \text { stability condition }
$$

The radius of the last stable configuration is thus determined by the condition

$$
\frac{\mathrm{d}}{\mathrm{d} a}(a \xi(a))=0, \quad \text { last stable state }
$$

Thus, if the average filling factor at the given radius $a$ is decreasing with the increase of radius faster than $1 / a$, there is stable shrinking of the adhesive contact. The critical configuration is determined by the condition that the filling factor is decreasing approximately as $1 / a$.

\section{Detachment of elongated shapes}

The rule that the last stable configuration approaches the incircle of the cross-section is not always applicable especially when the incircle is not defined uniquely. This can be easily illustrated on the example of a flatended stamp in form of a stretched rectangle (Fig. 10). As in the case of square shape, detachment starts at the sharp corners. However, we found the last stable configuration to correspond to the state when the detachments of two corners merge together forming a sort of "partial incircle" of the shape. Further increase of distance leads to an abrupt detachment (see Fig. 10(a)). 
(a)
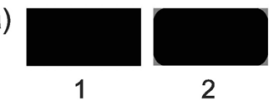

2

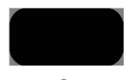

3

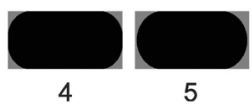

(b)

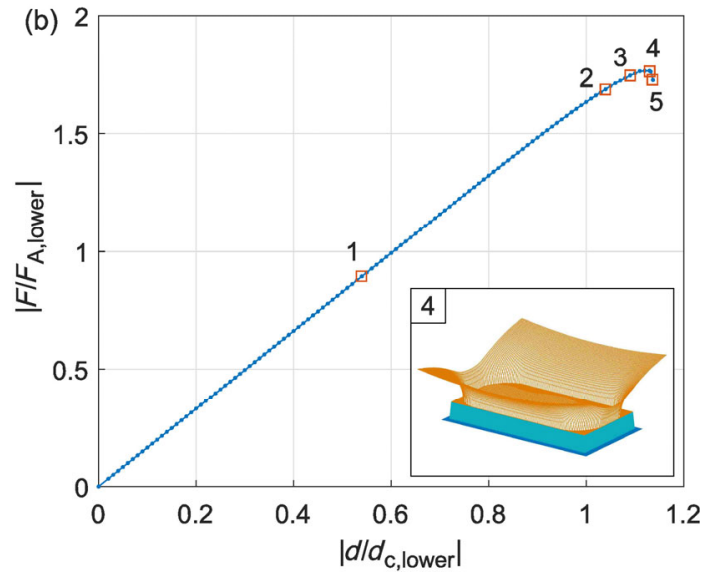

Fig. 10 Detachment of a stamp with a rectangular cross-section: (a) Consecutive shapes of the remaining contact area; the last one shown configuration corresponds to the last state of stable equilibrium. (b) The force-distance dependence. The complete detachment occurs in a configuration which strongly differs from the largest incircle of the rectangle.

It is interesting to note that this behavior can still be understood qualitatively within the above described concept of "damage filling factor". Let us consider the extreme case of a very long rectangular stamp as shown in Fig. 11. If the half width of the remaining adhering area is $a$, then the contact stiffness will scale approximately linearly with $a$ (up to a logarithmic

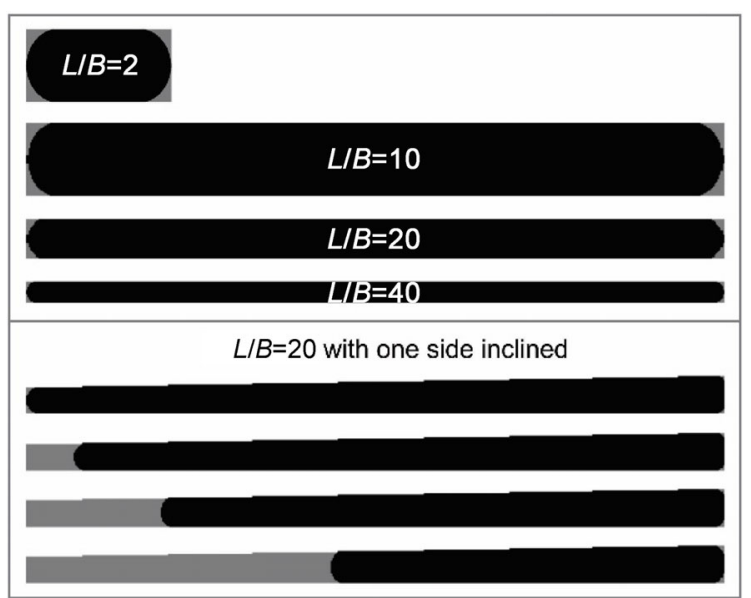

Fig. 11 Detachment of flat-ended stamps with rectangular crosssections having various aspect ratios $L / B$. We show only the final stable states. Independently from the aspect ratio, the final state corresponds to almost complete rectangle with rounded-up corners. When the shape has an inclination, a continuous detachment becomes possible. factor). On the other hand, as the thickness of the rectangle is constant, the "filling factor" will be approximately inversely proportional to the radius $a$. Thus, for the rectangle, the stability condition (30) is never satisfied. As a matter of fact, the rectangular shape represents a degenerate case when all configurations correspond to the critical state. Therefore, even a small slope of the shape leads to to the possibility of a stable propagation of the detachment front as illustrated in Fig. 11.

\section{Complicated contact shapes: Comparison of simulation and experiment}

In addition to our numerical studies, we conducted experiments with a series of compact flat indenter shapes. We did this to further validate our numerical method and to see whether the principal features of the detachment behavior could be reproduced. The experiments were conducted using a setup as depicted in Fig. 12. The rigid indenter consisted of a laser-cut acrylic glass with flat face. It was brought into contact with transparent gelatin with illumination from the sides. The acrylic glass was lifted with a precision linear stage attached to a strain gage sensor recording the adhesive force. The actual contact region was recorded from underneath using a digital camera.

In the distance-force-dependencies we find experimentally that the general features match those of the simulations. First we observe that the adhesive force always starts to rise linearly with the lifting height. In a second phase, detachment becomes visible and we observe a weaker rise of $F$ with $d$. When the lifting is stopped and reversed, we find that the contact configuration reassumes the shape corresponding to the same height from the lifting phase. Thus, we deal with reversible adhesion. Finally the contact is lost completely and relatively fast. It then consists of a quickly shrinking circle. As in the simulations, the last stable configuration can be roughly approximated by the largest circle to fit inside the initial shape.

Figure 13 presents a comparison of the detachment process from experiment and simulations. The consecutive shapes of the remaining adhesive contact zones are very similar to the simulation results for all experiments which we carried out. The differences 

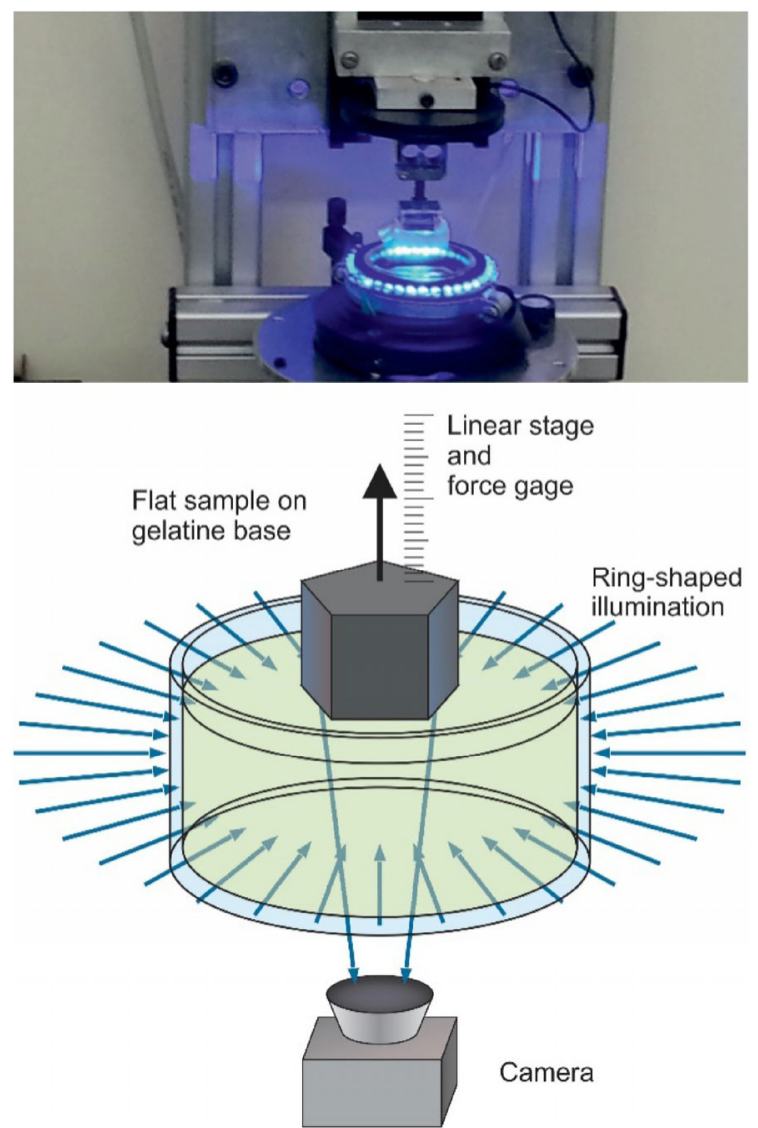

Fig. 12 Schematic display of the experimental setup. A flat but oddly shaped sample of acrylic glass is placed on a gelatin base and slowly pulled off. The contact between both can be observed by a camera through the gelatin. For increased visibility of the edges, the contact zone is illuminated in a circular fashion.

in the force-displacement relations are stronger: The plateau region, where most of the detachment happens, is larger in experiments than it is in simulations (see for instance the positions of the point $S_{1}, S_{2}$ and $S_{3}$ and the corresponding experimental points $E_{1}, E_{2}$ and $E_{3}$ in Fig. 13). This discrepancy may be related to the viscoelasticity of the highly deformable gelatin which we used in experiments. We noticed that changes of pull-off speed were directly altering the measured normal force when performed during the partial detachment phase. This hints to some time-depend response of the material and can explain the discrepancy with respect to $d$. Discrepancies between theory and experiment may also be due to the half-space approximation used in theoretical consideration. In the experiment, the finite size of the gelatin samples necessarily introduces deviations from the half-space assumption.
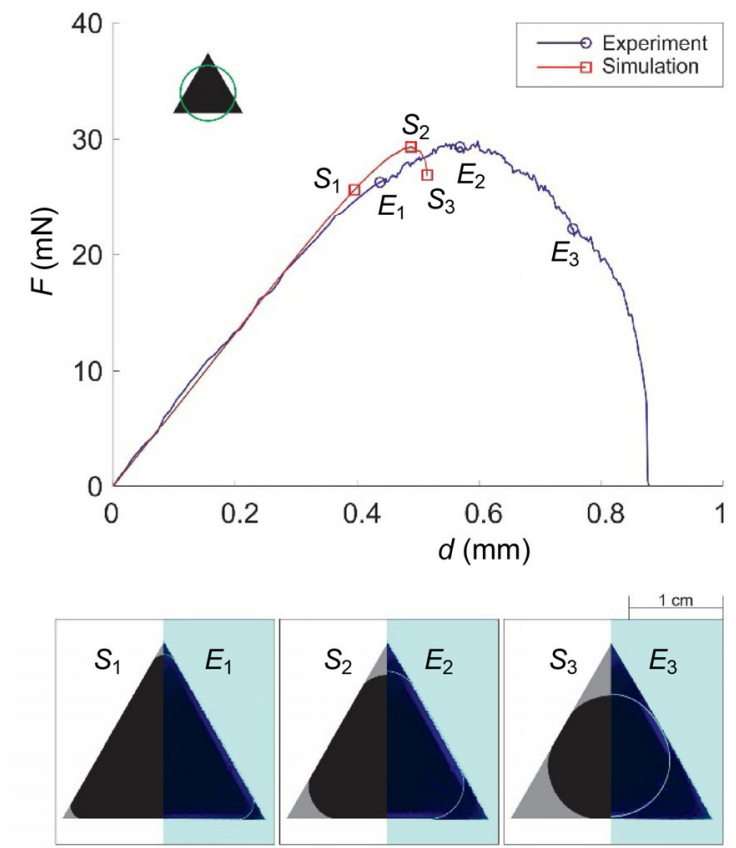

Fig. 13 Dependency of the attracting force on the pull-off distance for a triangular shape. The blue curve represents experimental data, while the red curve is obtained by simulation. The simulation results were fitted to the experimental data by assuming $E^{*}=6.02 \mathrm{kPa}$ and $\gamma_{12}=0.062 \mathrm{~N} \cdot \mathrm{m}^{-1}$. Highlighted points $\left(S_{1}, S_{2}, S_{3}, E_{1}, E_{2}, E_{3}\right)$ refer to Fig. 13 (below). The green circle in the shape representation depicts the equivalent Holm-radius of the triangular shape. In the lower part, the real contact area for selected pull-off-states is shown. All three pictures show numerical results on the left hand side, where the initial shape is gray, and remaining contact area is black. The right hand sides show photographic images of the contact. The edge of the contact zone can be seen as a bright line.

In spite of the differences in the force-distance dependencies, we consider the practically exact coincidence of the shapes of the consecutive contact forms as important experimental validation to the adhesive BEM formulation used in simulations.

Note that the presented experiments indicate that for the used gelatin the "stress criterion" of detachment as defined in Ref. [30] should be applied, not the "deformation criterion". According to the stress criterion, the same configurations of the adhesive contact are achieved at the same forces, not at the same distances.

The shapes shown in Figs. 13-15 are representative for the general behavior of the cases we investigated. First we observe $F$ to rise strictly linearly with $d$. In this phase, the contact area remains intact without any detachment and the ratio is equal to the contact stiffness of the initial shape. This is to be expected 

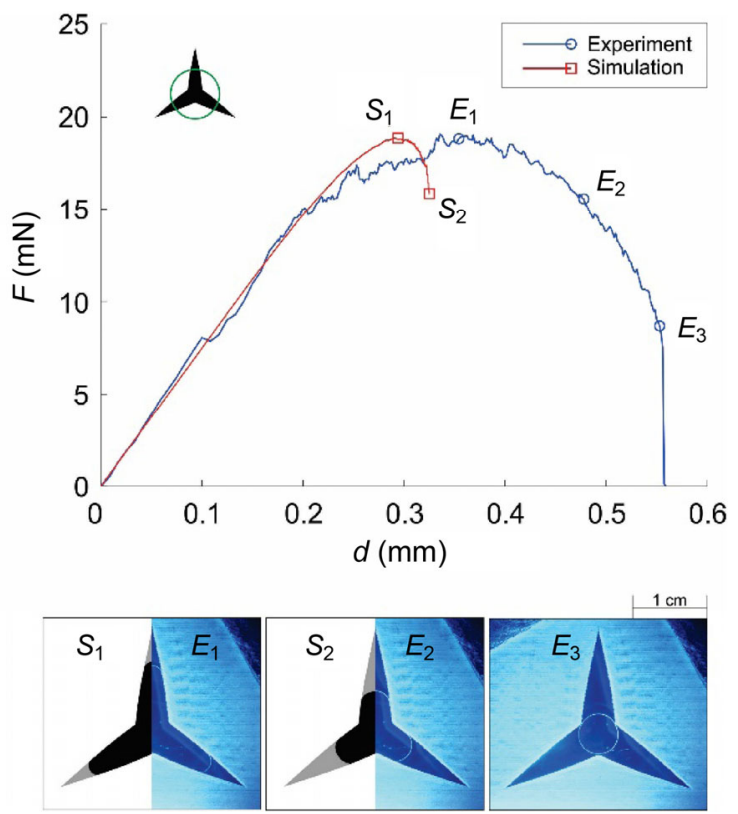

Fig. 14 Dependency of the attracting force on the pull-off distance for a star shape. The blue curve represents experimental data, while the red curve is obtained by simulation. The simulation results were fitted to the experimental data by assuming $E^{*}=7.60 \mathrm{kPa}$ and $\gamma_{12}=0.060 \mathrm{~N} \cdot \mathrm{m}^{-1}$. Highlighted points $\left(S_{1}, S_{2}, E_{1}, E_{2}, E_{3}\right)$ refer to the lower part where real contact area for selected pull-off-states are shown. For the third photograph of the experiment, no equivalent stable solution in the numerical simulation was reached.

from the linear elasticity. We then observe a transition to a second phase, where detachment starts at sharp corners or outstanding parts of the shape or border segments with high curvature and then moves inwards. In the $\bar{F}-\bar{d}$-curve, this goes along with a decline of the slope. After the bodies have lost contact at the sharp corners and outstanding parts, the region evolves in such a way, that the minimum radius of curvature is increased with increasing height. For various shapes, the force decreases after having reached a local maximum.

All simulations and experiments reached a point when the remaining contact collapses abruptly. The last stable contact region resembles the incircle of the initial contact zone. The run of the curve between phase 2 and the final detachment can have different characteristics. In the case of the triangular and star shape, we find a local maximum in the force. For a circle (not shown), the intermediate phase vanishes and the end of phase 1 coincides with the maximum force and the onset of final detachment. For more complicated shapes, the force can achieve several
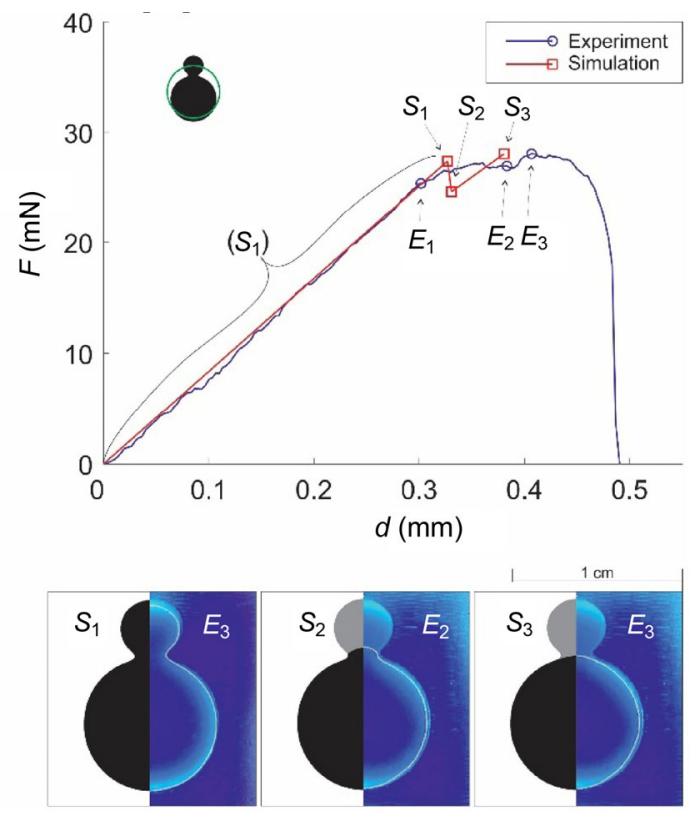

Fig. 15 Dependency of the attracting force on the pull-off distance for a shape composed of two circles. The blue curve represents experimental data, while the red curve is obtained by simulation. The simulation results were fitted to the experimental data by assuming $E^{*}=9.28 \mathrm{kPa}$ and $\gamma_{12}=0.030 \mathrm{~N} \cdot \mathrm{m}^{-1}$. Highlighted points $\left(S_{1}, S_{2}, S_{3}, E_{1}, E_{2}, E_{3}\right)$ refer to the lower part where the real contact area for selected pull-off-states is shown. The green circle in the shape representation depicts the equivalent Holm-radius of the shape. In the photographs of the experiment $\left(E_{1}, E_{2}, E_{3}\right)$, the actual edge of the contact has been retraced with a semi-transparent white line for better visibility.

maxima and have several jumps corresponding to partial instabilities.

The shape shown in Fig. 15 was designed to cause a discontinuity in the adhesive force when the smaller circle detaches and we obtain two local maxima in the $\bar{F}-\bar{d}$-curve. The reason for this is the constriction in the shape. According to the discussion in Section 8, a stable shrinking of the contact area is only possible if the filling factor $\xi$ is increasing fast enough with decreasing the radius. In the case of shapes with constriction, we have the opposite case when the filling factor decreases with decreasing the radius. Thus, the propagation of the contact will be instable and there will be a jump to the next state when the condition of continuous shrinking, Eq. (30), is satisfied again.

Note however, that there are some differences between experiment and theory: In the simulation, the detachment of the upper part from $S_{1}$ to $S_{2}$ is abrupt. While the experiment also shows the feature of 
non-monotonous dependency of force as a function of distance, the dependency has no abrupt stages. The reason could be again the viscoelasticity of the gelatin.

Also note that in the experimental state $E_{3}$ shown in Fig. 15, the boundary line of the adhesive zone does not match exactly the contour of the stamp. This may indicate that the assumption of infinitely short action range of adhesive forces lying behind the JKRtype of adhesion is not completely applicable to gelatin. Small detachment at the boundary is an effect which can be expected in the case of finite action range of the adhesion forces [31].

\section{Adhesion of graded media}

In the previous sections, we studied the adhesive contact of flat punches that were placed in contact with a linear elastic medium. We shall now consider the adhesive problem when the elastic body is characterized by a depth-dependent modulus of elasticity. This socalled functionally graded materials (FGM) became increasingly popular since 1990s. The gradually varying composition and structure of FGM result in the continuous changes in properties of materials, thus providing new mechanical properties which cannot be achieved with homogeneous materials [31, 32]. Living species have "discovered" FGM millions of years ago. Gradient media can be found in many biological structures as skin, bones or bamboo trees [33].

In the present section we only consider materials whose elastic coefficient is a function of the normal coordinate $E=E(z)$. This dependence can be either stepwise (as, e.g., in layered or coated materials) or continuous (FGM). For simplicity, we confine ourselves to the model case of materials with a power-law dependency of the elastic modulus on depth:

$$
E(z)=E_{0}\left(\frac{z}{c_{0}}\right)^{k},-1<k<1
$$

where $E_{0}$ is a characteristic elastic modulus and $c_{0}$ is a characteristic length. We additionally assume that the Poisson ratio of the graded medium is constant and equal to

$$
v=\frac{1}{2+k}
$$

which guarantees decoupling of the normal and tangential contact problems [34].

For the graded medium defined by Eqs. (32) and (33), the contact stiffness for indentation with a rigid cylinder having the radius $a$ is given by Refs. [34, 35]

$$
k(a)=\frac{4(2+k) \cos (k \pi / 2)}{(3+k)} \frac{E_{0}}{c_{0}^{k}} a^{1+k}
$$

For the total energy $\frac{1}{2} k(a) d^{2}-\gamma_{12} \pi a^{2}$, we thus have

$$
U_{\text {tot }}=\frac{2(2+k) \cos (k \pi / 2)}{(3+k)} \frac{E_{0}}{c_{0}^{k}} a^{1+k} d^{2}-\gamma_{12} \pi a^{2}
$$

For $k<1$, the total energy has only one maximum at the critical radius $a_{\mathrm{c}}$ determined by the condition

$$
\begin{aligned}
& \frac{\partial U_{\mathrm{tot}}}{\partial a}=\frac{2(2+k)(1+k) \cos (k \pi / 2)}{(3+k)} \frac{E_{0}}{c_{0}^{k}} a^{k} d^{2}-\gamma_{12} 2 \pi a \\
& \frac{\partial U_{\mathrm{tot}}}{\partial a}\left(a=a_{c}\right)=0
\end{aligned}
$$

As in the case of homogeneous media discussed in Section 2, the contact either shrinks to zero or expands to infinity depending on whether the initial radius was smaller or larger than $a_{\mathrm{c}}$. For a finite indenter, Eq. (36) determines the relation between the critical detachment distance and the radius of the indenter:

$$
\frac{2(2+k)(1+k) \cos (k \pi / 2)}{(3+k)} \frac{E_{0}}{c_{0}^{k}} a^{k} d_{c}^{2}=\gamma_{12} 2 \pi a
$$

For the critical distance we get

$$
d_{\mathrm{c}}=\left(\frac{2 \pi(3+k) \gamma_{12} c_{0}^{k}}{2(2+k)(1+k) \cos (k \pi / 2) E_{0} a^{k-1}}\right)^{1 / 2}
$$

and for the adhesion force $F_{\mathrm{A}}=k(a) d_{\mathrm{c}}$,

$$
F_{\mathrm{A}}=\sqrt{\lambda(k) 8 \pi E_{0} \gamma_{12} \frac{a^{3+k}}{c_{0}^{k}}}
$$

with

$$
\lambda(k)=\frac{2(2+k) \cos (k \pi / 2)}{(3+k)(1+k)}
$$

For damaged surfaces with the average filling factor $\xi(a)$, the adhesion force is estimated similarly to the 
procedure described by Eqs. (25)-(28) and is roughly equal to

$$
F_{\mathrm{A}} \approx \sqrt{\lambda(k) 8 \pi E_{0} \xi(a) \gamma_{12} \frac{a^{3+k}}{c_{0}^{k}}}
$$

A numerical procedure for the simulation of graded materials can be formulated using the same idea of balancing elastic energy and surface energy as described in Section 3. A detailed analysis carried out in Ref. [28] shows that now the detachment criterion (22) has to be replaced by

$$
\sigma_{\mathrm{c}}=\sqrt{\Psi(k, v) \frac{E_{0} \gamma_{12}}{c_{0}^{k} \Delta^{1-k}}}
$$

where $\Psi(k, v)$ is a factor depending on the power $k$ and the Poisson ratio $v$, which was found numerically in Ref. [28]. An example of the detachment of a flatended indenter having the shape of a star calculated with BEM using the detachment criterion (42) is shown in Fig. 16.

(a)

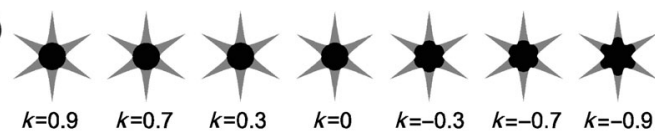

(b)

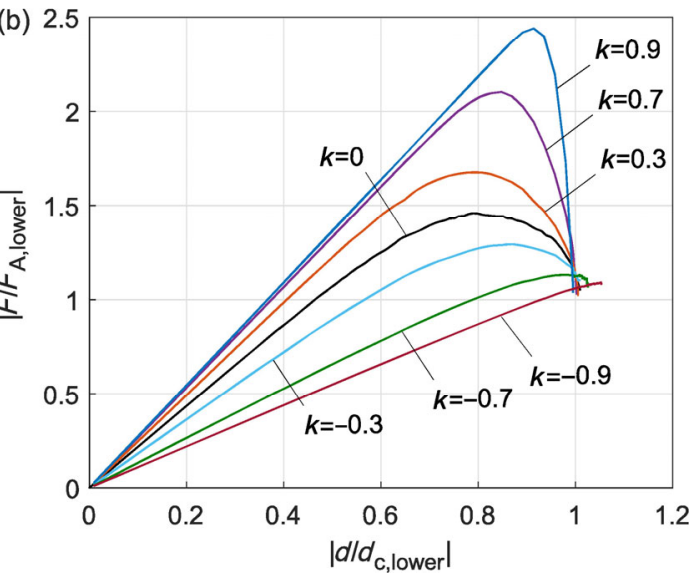

Fig. 16 Detachment of a flat-ended stamp with cross-section in form of a star from gradient media with various power $k$. (a) The last stable configuration for different $-1<k<1$. (b) The forcedisplacement dependencies show that the media with positive power $k$ develop a much more pronounced pre-detachment leading to a maximum of force, while the media with large negative powers tend to a "brittle" detachment almost without pre-detachment. In the limiting case $k=-1$ the force-displacement dependence degenerates into a linear dependency up to the point where the contact suddenly is lost.
For all media with $0<k<1$, the detachment starts at the sharp ends on the outside and ends up at the incircle of the star. The larger the power of the medium (thus the softer it is at the immediate surface) the larger is the maximum force and the work of fracture after achieving the maximum force (fracture toughness) contact. Media with negative power $-1<k<0$ show a different behavior. Now the last stable state is reached earlier than the incircle of the shape would suggest. For the limiting case $k=-1$ the last configuration is almost the non-disturbed initial shape. Correspondingly, the deviation from the force-displacement linearity decreases with increasing absolute value of $k$ and the fracture toughness decreases rapidly. Thus, the soft surface facilitates both the magnitude of the adhesion force and the work of adhesion between maximum force and complete collapse of the contact.

\section{Discussion and conclusion}

As expected from our theoretical considerations, the adhesive contact of flat bodies within the assumptions of JKR theory can clearly depend on the macroscopic shape of the contact zone. Our results show that adhesion is not only governed by small-scale roughness properties of the surfaces but depends strongly on the bulk shape of the initial adhesive zone. During pull-off, the detachment starts at the exposed outer parts of the shape such as tentacles or sharp corners, then the contact zone shrinks, approaching a final state after which the detachment occurs in an instable way. In the final state, the remaining contact has a shape with circularly rounded corners. In the course of detachment, one or multiple maxima can occur. These phenomena can be observed both in experiment and simulation. In direct comparison however, the experiments develop more slowly near the instabilities.

While the macroscopic shape of the stamp has essential influence on the adhesive strength, small inner discontinuities do not. Their influence can be characterized relatively well with a filling parameter quantifying the fraction of the continuous part of the cross-section relatively to the nominal area. In particular, the detachment of square brushes occurs almost in the same way as of the corresponding full square. For determining the adhesive force, only the 
work of adhesion has to be multiplied with the filling factor of the shape.

Special attention has to be paid, when the filling factor depends on the distance from the center of the contact zone. This dependence will then be the parameter determining both the character of detachment and the size and shape of the final state before sudden loss of contact.

We anticipate our work to inspire a broader view on adhesion problems, differentiating small-scale roughness effects from the macro-shape. For instance, one could design adhesive bonds that show distinct partial detachment below their maximum strength, giving an early warning of critical failure.

\section{Acknowledgement}

Authors acknowledge the assistance of C. Jahnke in conduction of experiments and very useful discussions of adhesion with gradient media with M. Heß and E. Willert. This work has been conducted under partial financial support from DFG (Grant number PO 810/22-1).

Contributions of authors: R. Pohrt built the experimental setup and processed the experimental data. R. Pohrt and Q. Li executed the numerical simulations. Theoretical analysis was carried our primarily by V. L. Popov. All authors contributed equally to the writing of the manuscript.

Open Access: The articles published in this journal are distributed under the terms of the Creative Commons Attribution 4.0 International License (http:// creativecommons.org/licenses/by/4.0/), which permits unrestricted use, distribution, and reproduction in any medium, provided you give appropriate credit to the original author(s) and the source, provide a link to the Creative Commons license, and indicate if changes were made.

\section{References}

[1] Lee L H (Ed.). Fundamentals of Adhesion. New York: Springer Science \& Business Media, 1991

[2] Dzyaloshinskii I E, Lifshitz E M, Pitaevskii L P. General Theory of van der Waals' Forces. Soviet Physics Uspekhi 4: 153-176 (1961)
[3] Landau L D, Lifshitz E M. Statistical Physics, Pt. 2, (Volume 9 of the Course of Theoretical Physics). Oxford: Pergamon Press, 1980

[4] Afferrante L, Carbone G. The ultratough peeling of elastic tapes from viscoelastic substrates. Journal of the Mechanics and Physics of Solids 96: 223-234 (2016)

[5] Popov V L, Filippov A E, Gorb S N. Biological microstructures with high adhesion and friction. Numerical approach. Physics-Uspekhi 59(9): 829-845 (2016)

[6] Autumn K, Liang Y A, Tonia Hsieh S, Zesch W, Chan W P, Kenny T W, Fearing R, Full R J. Adhesive force of a single gecko foot-hair. Nature 405: 681-685 (2000)

[7] Köster S, Janshoff A. Editorial-Special issue on mechanobiology. Biochimica et Biophysica Acta (BBA) - Molecular Cell Research 1853(11, Part B): 2975-2976 (2015)

[8] Popov V L. Contact Mechanics and Friction-Physical Principles and Applications. Berlin: Springer-Verlag Berlin Heidelberg, 2010

[9] Kendall K. Molecular Adhesion and Its Applications. New York (US): Springer Science \& Business Media, 2001

[10] Luan B, Robbins M O. The breakdown of continuum models for mechanical contacts. Nature 435(7044): 929-932 (2005)

[11] Ciavarella M. On Pastewka and Robbins' criterion for macroscopic adhesion of rough surfaces. Journal of Tribology 139(3): 031404 (2017)

[12] Guduru P R. Detachment of a rigid solid from an elastic wavy surface: theory. Journal of the Mechanics and Physics of Solids 55(3): 445-472 (2007)

[13] Johnson K L, Kendall K, Roberts A D. Surface energy and the contact of elastic solids. Proc. R. Soc. London A 324: 301-313 (1971)

[14] Maugis D. Adhesion of spheres: The JKR-DMT transition using a Dugdale model. Journal of Colloid and Interface Science 150(1): 243-269 (1992)

[15] Cheng A H-D, Cheng D T. Heritage and early history of the boundary element method. Engineering Analysis with Boundary Elements 29: 268-302 (2005)

[16] Cruse T A. Boundary Element Analysis in Computational Fracture Mechanics. Kluwer, Dordrecht, 1988

[17] Blandford G E, Ingraffea A R, Liggett J A. Two-dimensional stress intensity factor computations using the boundary element method. International Journal for Numerical Methods in Engineering 17(3): 387-404 (1974)

[18] Pohrt R, Popov V L. Adhesive contact simulation of elastic solids using local mesh-dependent detachment criterion in Boundary Elements Method. Facta Universitatis, Series: Mechanical Engineering 13(1): 3-10 (2015)

[19] Hulikal S, Bhattacharya K, Lapusta N. A threshold-force 
model for adhesion and mode I fracture. arXiv:1606.03166.

[20] Rey V, Anciaux G, Molinari J-F. Normal adhesive contact on rough surfaces: efficient algorithm for FFT-based BEM resolution. Comput Mech, DOI: 10.1007/s00466-017-1392-5 (2017)

[21] Kendall K. The adhesion and surface energy of elastic solids. Journal of Physics D: Applied Physics 4(8): 1186 (1971)

[22] Li Q, Popov V L. Indentation of flat-ended and tapered indenters with polygonal cross-section. Facta Universitatis Series: Mechanial Engineeering 14(3): 241-249 (2016)

[23] Holm R, Holm E. Electric Contacts Handbook. Berlin: Springer-Verlag, 1958

[24] Griffith A A. The phenomena of rupture and flow in solids. Philosophical Transactions of the Royal Society of London, A 221: 163-198 (1921)

[25] Pohrt R, Li Q. Complete boundary element formulation for normal and tangential contact problems. Physical Mesomechanics 17(4): 334-340 (2014)

[26] Putignano C, Afferrante L, Carbone G, Demelio G. A new efficient numerical method for contact mechanics of rough surfaces. International Journal of Solids and Structures 49(2): 338-343 (2012)

[27] Maugis D, Barquins M. Adhesive contact of a conical punch on an elastic half-space. Le Journal de Physique Lettres 42(5): 95-97 (1981)

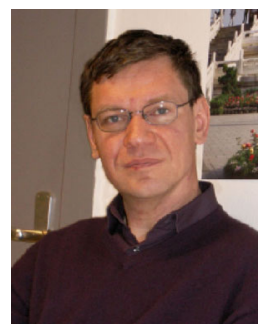

Valentin L. POPOV. He is full professor at the Berlin University of Technology. He studied physics and obtained his doctorate in 1985 from the Moscow State Lomonosov University. 1985-1998 he worked at the Institute of Strength Physics and Materials Science of the Russian Academy of Sciences and was a guest professor in the field of theoretical physics at the University of Paderborn (Germany) from 1999 to 2002. Since 2002 he is the head of the Department of System Dynamics and the Physics of Friction at the Berlin University of Technology. He has published over 300 papers in leading international journals and is the author of the book "Contact Mechanics and Friction: Physical principles and applications" which appeared in three editions in
[28] Li Q, Popov V L. Boundary element method for normal non-adhesive and adhesive contacts of power-law graded elastic materials. arXiv:1612.08395 (2016)

[29] Argatov I I, Li Q, Pohrt R, Popov V L. Johnson-KendallRoberts Adhesive Contact for a Toroidal Indenter. Proceedings of the Royal Society of London, Series A 472(2191): (2016)

[30] Popov V L. Basic ideas and applications of the method of reduction of dimensionality in contact mechanics. Physical Mesomechanics 15: 254-263 (2012)

[31] Maugis D. Adhesion of spheres: The JKR-DMT transition using a Dugdale model. Journal of Colloid and Interface Science 150(1): 243-269 (1992)

[32] Suresh S. Graded materials for resistance to contact deformation and damage. Science 292: 2447-2451 (2001)

[33] Jha D K, Kant T, Singh R K. A critical review of recent research on functionally graded plates. Composite Structures 96: 833-849 (2013)

[34] Hess M, Popov V L. Method of dimensionality reduction in contact mechanics and friction: A user's handbook. II Powerlaw graded materials. Facta Universitatis, Series: Mechanical Engineering 14(3): 251-268 (2016)

[35] Heß M. A simple method for solving adhesive and nonadhesive axisymmetric contact problems of elastically graded materials. International Journal of Engineering Science 104: 20-33 (2016)

German, English, Chinese, and Russian. He is the member of editorial boards of many international journals and is organizer of more than 20 international conferences and workshops over diverse tribological themes. Prof. Popov is Honorary Professor of the Tomsk Polytechnic University, of the East China University of Science and Technology, and of the Changchun University of Science and Technology and Distinguished Guest Professor of the Tsinghua University. His areas of interest include tribology, nanotribology, tribology at low temperatures, biotribology, the influence of friction through ultrasound, numerical simulation of contact and friction, research regarding earthquakes, as well as topics related to materials science such as the mechanics of elastoplastic media with microstructures, strength of metals and alloys, and shape memory alloys. 


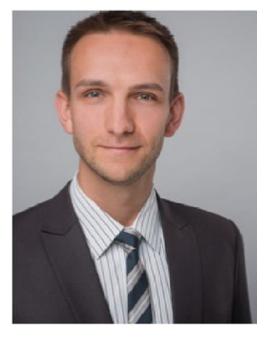

Roman POHRT. He is independent researcher at the Berlin University of Technology. He studied physical engineering science with special focus on simulation and optimization of discrete and continuous problems. Since he joined the group of Prof. V. Popov in 2010, he has been conducting experimental and numerical research on a variety of tribology related industry problems. In his PhD thesis R. Pohrt

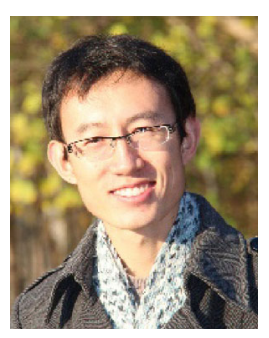

Qiang LI. He is a postdoctoral researcher at the Berlin University of Technology. He studied mechanical engineering in East China University of Science and Technology. He obtained his doctorate at the Berlin University of Technology in 2014 and now works as a scientific researcher at the focussed on linking scales in the elastic contact of fractal rough surfaces, for which he was awarded by the German Tribological Society in 2013. R. Pohrt has authored a series of influential papers on different tribological problems, applying and extending stateof-the-art numerical methods. His areas of interest include contact mechanics, rail-wheel-interaction of trains, manufacturing technology, and lubrication and more generally the influence of surface topography on tribological phenomena.

Department of System Dynamics and the Physics of Friction headed by Prof. V. L. Popov. He has published over 20 papers in international journals including Physical Review Letters. His scientific interests include tribology, elastomer friction, hydrodynamic lubricated contact, numerical simulation of frictional behaviors, and fast numerical method based on boundary element method. 